\title{
THE EXPONENT OF CONVERGENCE OF RIEMANN SURFACES. BASS RIEMANN SURFACES
}

\author{
J.L. Fernández* and J.M. Rodríguez
}

\section{Introduction}

In this paper we study the exponent of convergence of Riemann surfaces and, especially, its behaviour under quasiconformal mappings.

A Riemann surface is Green if it possesses non-constant positive superharmonic functions or, equivalently, a Green's function. It is well known that if two Riemann surfaces are quasiconformally equivalent and, one is Green, the other is Green too ([Pf]). In [Ro] Royden asked if the same result holds for the Liouville property: i.e., not having non-constant bounded harmonic function. For plane domains being Green and not satisfying Liouville's property is the same. Not so for higher genus ([A-S, p. 256], [T1] and [T2]). A few years ago P. Doyle and T. Lyons ([L]) independently found pairs of quasiisometric (a fortiori quasiconformally equivalent) Riemann surfaces such that one has Liouville's property and the other does not.

A basic conformal invariant of a hyperbolic Riemann surface $S$ is the bottom of the spectrum of the Laplace-Beltrami operator, $b(S)$. This can be defined in terms of Rayleigh's quotient as

$$
b(S)=\inf _{\varphi \in C_{c}^{\infty}(S)} \frac{\iint\|\nabla \varphi\|^{2} d \omega}{\iint \varphi^{2} d \omega}
$$

where \|\|$, \nabla$ and $d \omega$ refer to the Poincaré metric of $S$. (We assume here and hereafter that the universal cover of $S$ is the unit disk $\Delta$ or, equivalently, the upper halfplane $U$ in $\mathbf{C}$; that is what the adjective hyperbolic refers to. From now on all Riemann surfaces considered will be hyperbolic. The Poincaré metric of $\Delta$ is $d s=2|d z| /\left(1-|z|^{2}\right)$ and the Poincaré metric of $S$ is the unique metric in $S$ such that the universal covering map is a local isometry.)

We remark that the Dirichlet integral is a conformal invariant; it follows that if $S=\Omega \subset \mathbf{C}$, then

$$
\iint_{\Omega}\|\nabla \varphi\|^{2} d \omega=\iint_{\Omega}|\nabla \varphi|^{2} d x d y
$$

* Research supported by a grant of CICYT, Ministrio de Educación y Ciencia, Madrid, Spain.

doi:10.5186/aasfm.1990.1510 
where ||$, \nabla$ and $d x d y$ refer to the Euclidean metric in the second integral.

The number $b$ belongs to the interval $\left[0, \frac{1}{4}\right]$. Actually, a theorem of ElstrodtPatterson-Sullivan says that

$$
b(S)= \begin{cases}\frac{1}{4} & \text { if } 0 \leq \delta(S) \leq \frac{1}{2}, \\ \delta(S)(1-\delta(S)) & \text { if } \frac{1}{2} \leq \delta(S) \leq 1\end{cases}
$$

where $\delta(S)$ is the exponent of convergence of $S$ ([S, p. 333]).

A Riemann surface is termed bass if $b(S)=0$, or equivalently, $\delta(S)=1$. If a Riemann surface has $b(S)>0$, then it has a Green's function; in fact there is a $C^{\infty}$ positive eigenfunction of the Laplace-Betrami operator satisfying

$$
\Delta \psi=t \psi
$$

for every $t<b(S)$. See, e.g., [S, p. 328].

Our sign convention for $\Delta$ is such that $-\partial^{2} / \partial \theta^{2}=\Delta$ in the case of the circle. So $\Delta$ is a positive operator.

We shall prove:

Theorem 1. If a Riemann surface $S_{1}$ is bass and is quasiconformally equivalent to $S_{2}$ then $S_{2}$ is bass.

Thus being bass is a quasiconformally invariant property.

It is easy to see that if $f: S_{1} \rightarrow S_{2}$ is $k$-quasiconformal then $k^{-1} \delta\left(S_{1}\right) \leq$ $\delta\left(S_{2}\right) \leq k \delta\left(S_{1}\right)$, but, of course, this does not help in the proof of Theorem 1 .

We shall see that being bass is a geometrical concept.

We shall say that a Riemann surface $S$ satisfies the hyperbolic isoperimetric inequality (HII) if there exists a constant $h(S)>0$ so that for every relatively compact open set $G$ with smooth boundary one has

$$
A_{S}(G) \leq h(S) L_{S}(\partial G) .
$$

Here and hereafter, $A_{S}$ and $L_{S}$ refer to Poincaré area and length of $S$. Domains $G$ as above will be said to belong to $\mathcal{D}(S)$.

Of course, the hyperbolic plane satisfies the HII with $h=1$.

A general result of Cheeger says that if a Riemann surface $S$ satisfies HII then it is not bass and actually, $b(S) \geq 1 / 4 h(S)^{2}$. It turns out that negative curvature forces an inequality in the opposite direction. Namely:

Theorem 2. A Riemann surface $S$ is not bass if and only if it satisfies HII. Moreover, for an absolute constant $C$, we have

$$
\frac{1}{4} \leq b(S) h(S)^{2} \quad \text { and } \quad b(S) h(S) \leq C<\frac{3}{2} .
$$


This result is well known. It also holds in higher dimensions and with weaker assumptions on curvature, see [B, p. 228]. But our proof is direct in our situation and the argument we use is needed for Theorem 1.

Next, we move on to study which plane domains are bass. Green plane domains are those whose complements has positive logarithmic capacity. Deciding when a plane domain is bass is more delicate. For instance, $\Delta-\{0\}-\left\{1 / 2^{n}\right\}_{n=1}^{\infty}$ is bass while $\Delta-\left\{1-1 / 2^{n}\right\}_{n=1}^{\infty}$ is not.

We do have a necessary condition and a sufficient condition for a plane domain to be bass which are quite close.

A domain $G$ in the sphere is called modulated if there is an upper bound for the modulus of every doubly connected domain $H \subset G$ which separates the boundary of $G$. The lowest such upper bound is called the modulus of $G$.

There is a number of characterizations and known properties of these domains. (See e.g. [B-P], [Po1], [Po2], [M]).

Theorem 3. Assume that $G$ is modulated and that $\left\{a_{n}\right\}_{n=1}^{\infty}$ is a separated sequence in $G$, i.e.

$$
\inf _{n \neq m} d_{G}\left(a_{n}, a_{m}\right)>0 .
$$

Then the domain $\hat{G}=G-\left\{a_{n}\right\}_{n=1}^{\infty}$ is not bass.

Here, and hereafter, $d_{G}$ means Poincaré distance in $G$.

In $[\mathrm{F} 1]$ it was shown that modulated domains are not bass.

Let $B$ a compact set in the complex plane.

If $p$ belongs to $B$, we define for $r, 0<r<\operatorname{diam} B$,

$$
\alpha(p, r)=\operatorname{cap}(\bar{\Delta}(p, r) \cap B) r
$$

and

$$
\beta(p, r)=\frac{1}{r} \inf \{s: \Delta(p, s) \cap B \supset \Delta(p, r) \cap B\} .
$$

If $H$ is a plane domain and $\infty$ belongs to $H$, it is known, [Po1, p. 192 and 193], [Po2, p. 302 and 307], that $H$ is modulated if and only if $\inf _{p, r} \alpha(p, r)>0$ and also that $H$ is modulated if and only if $\inf _{p, r} \beta(p, r)>0$, where in both instances the set $B$ involved is $B=\partial H$. Notice that $\alpha(p, r) \leq \beta(p, r)$. A set $B$ with $\inf \{\alpha(p, r): p \in B, 0<r<\operatorname{diam} B\}>0$ is called uniformly perfect.

We have the following converse of Theorem 3 .

Theorem 4. Assume that $H$ is a plane domain which is not bass and $\infty \in H$. Then $\hat{\mathbf{C}} \backslash H$ is a disjoint union $P \cup I$, where $I$ is the set of all isolated points of $\partial H$. The points of $I$ are separated in $\hat{\mathbf{C}} \backslash P=H \cup I$ and there exist constants $c_{1}$ and $c_{2}$ so that if $p \in P$ then

$$
\beta(p, r) \geq c_{1}
$$


or

$$
\alpha(p, r \beta(p, r)) \geq c_{2}
$$

where $\alpha$ and $\beta$ are as above with $B$ replaced by $P$.

Also $\operatorname{cap}(\Delta(p, r) \cap P)>0$ for each $p \in P$, for each $r>0$.

We have examples showing that the condition of Theorem 3 is not necessary while that of Theorem 4 is not sufficient.

Notation. By $C$ we will mean an absolute constant which can change its value from line to line, and even in the same line.

If $\Omega$ is a plane domain, $\lambda_{\Omega}$ means the density of the Poincaré metric in $\Omega, d$ means Euclidean distance in $\mathbf{C}, \Delta(a, r)$ is the Euclidean open disk with centre $a$ and radius $r, \Delta(a, r)^{*}=\Delta(a, r)-\{a\}, \Delta_{r}=\Delta(0, r)$ and $\Delta_{1}=\Delta$.

If $F$ is a closed set $F^{i}=\operatorname{iso}(F)$ means the set of all isolated points of $F$, and $F^{d}=\operatorname{der}(F)$ means the set of all accumulation points of $F$ (the derived set of $F$ ) and $\operatorname{cap}(F)$ denotes the logarithmic capacity of the set $F$.

The organization of the paper is as follows. In Section 1 we prove Theorem 2. The proof of Theorem 1 appears in Section 2, and finally Theorems 3 and 4 are dealt with in Section 3 and 4, respectively. Section 5 contains some remarks.

\section{Proof of Theorem 2}

If $S$ satisfies HII then as we have already remarked it follows from Cheeger's inequality ([Che], [Cha, p. 95]) that $S$ is not bass and, in fact,

$$
b(S) h(S)^{2} \geq \frac{1}{4}
$$

Let us assume that $S$ is not bass and we shall see that $S$ satisfies HII.

Our first step is to verify that we only have to check that HII holds for geodesic domains in $S$. By a geodesic domain we mean a domain $G \subset S$, such that $\partial G$ consist of finitely many closed simple geodesics, and $A_{S}(G)$ is finite. $G$ does not have to be relatively compact since it may "surround" finitely many punctures. Thus we may consider punctures as non-proper closed geodesics of zero length. We first dispose of some elementary cases.

Lemma 1.1. a) If $S$ is a simply or doubly connected Riemann surface then HII holds with constant 1 .

b) If $S$ is a Riemann surface and $\Omega \in \mathcal{D}(S)$ is simply or doubly connected then

$$
A_{S}(\Omega) \leq L_{S}(\partial \Omega)
$$

Part a) is elementary and part b) follows from a).

Lemma 1.2. Assume that $S$ satisfies HII for geodesic subdomains; then $S$ satisfies HII. 
Proof. Assume that $\Omega \in \mathcal{D}(S)$ and that

$$
L_{S}(\partial \Omega)<\varepsilon A_{S}(\Omega)
$$

where $\varepsilon<\frac{1}{2}$. We shall see that there is a geodesic subdomain $G$ with

$$
L_{S}(\partial G)<\frac{\varepsilon}{1-2 \varepsilon} A_{S}(G) .
$$

This will finish the proof.

Because of Lemma 1.1 we may assume that $\partial \Omega$ consists of finitely many closed simple disjoints curves $\gamma_{1}, \ldots, \gamma_{n}$, so that $\gamma_{i}, \gamma_{j}$ are not freely homotopic if $i \neq j$, and $\gamma_{i}$ is not homotopic to 0 .

Let $b_{i}$ be the geodesic in the homotopy class of $\gamma_{i}$ (recall that $b_{i}$ could be a puncture). Let $G$ be the domain "bounded" by the $b_{i}$. Then $G$ is a geodesic domain, and $L_{S}(\partial G) \leq L_{S}(\partial \Omega)$. Now we have to compare $A_{S}(G)$ with $A_{S}(\Omega)$ and in fact we will see that

$$
A_{S}(G)>A_{S}(\Omega)-2 L_{S}(\partial \Omega)
$$

When we replace a $\gamma_{i}$ by a $b_{i}$ we loose at most an area of $L_{S}\left(\gamma_{i}\right)+L_{S}\left(b_{i}\right)$. This follows, if $\gamma_{i} \cap b_{i}=\emptyset$, from Lemma $1.1 \mathrm{~b}$ ) since they bound a doubly connected domain, and if $\gamma_{i} \cap b_{i} \neq \emptyset$, then we lift to the unit disk and use the HII there to obtain the inequality. If $b_{i}$ is a puncture, we actually gain area.

From (1.1) and (1.3) we obtain

$$
L_{S}(\partial \Omega) \leq \frac{\varepsilon}{1-2 \varepsilon} A_{S}(G)
$$

and since $L_{S}(\partial G) \leq L_{S}(\partial \Omega)$ we obtain the result.

Moreover, if we have $A_{S}(G) \leq h_{g}(S) L_{S}(\partial G)$ for every geodesic domain $G$, then

$$
h(S) \leq 2+h_{g}(S)
$$

We need information about geodesics and punctures, which we record in the following lemmas.

Lemma 1.3. Let $S$ be a Riemann surface and $\gamma$ a closed simple geodesic in $S$. Let $\Omega$ be $\Omega=\left\{p \in S: d_{S}(p, \gamma) \leq d\right\}$. Then

$$
A_{S}(\Omega) \leq 2 \sinh (d) \cdot L_{S}(\gamma)
$$

The inequality is sharp. 
Proof. Let $T$ be the Möbius transformation from $U$ onto $U$ representing $\gamma$. We may assume, since this can be achieved by conjugation, that $T z=\lambda z$ with translation length $\log \lambda=L_{S}(\gamma)$, and $\pi$ (the universal covering map) maps

$$
\tilde{\gamma}=\left\{i y: 1 \leq y<e^{L_{S}(\gamma)}\right\}
$$

onto $\gamma$.

If $\tilde{\Omega}=\left\{z \in U: d_{U}(z, \tilde{\gamma}) \leq d, 1 \leq|z| \leq e^{L_{S}(\gamma)}\right\}$, then $\pi(\tilde{\Omega})=\Omega$ and $A_{U}(\tilde{\Omega})=2 \sinh (d) \cdot L_{U}(\tilde{\gamma})=2 \sinh (d) \cdot L_{S}(\gamma)$.

Now,

$$
A_{S}(\Omega) \leq A_{U}(\tilde{\Omega})=2 \sinh (d) \cdot L_{S}(\gamma)
$$

where the inequality follows because holomorphic mappings do decrease area, by Schwarz's lemma.

Let $E_{1}, E_{2}$ be nonvoid disjoint sets on the Riemann surface $S$ and denote by $\Gamma$ the family of connected arcs which join $E_{1}$ and $E_{2}$. We write $\lambda_{S}(\Gamma)=$ $\lambda_{S}\left(E_{1}, E_{2}\right)$ and call this quantity the extremal distance of $E_{1}$ and $E_{2}$ relatively to $S$, where $\lambda_{S}(\Gamma)$ is the extremal length of $\Gamma$ in $S$ (for details see [A-S, p. 220-225]).

If $S$ is the interior of a compact bordered surface $\bar{S}$ and $E_{1}, E_{2}$ consist of a finite number of arcs or full contours on the border, then there exists a unique bounded harmonic function $u$ which is 0 on $E_{1}, 1$ on $E_{2}$, and whose normal derivative vanishes on the remaining part of the border.

Theorem ([A-S, p. 225]). The extremal distance between $E_{1}$ and $E_{2}$ is equal to $1 / D(u)$.

We recall that the extremal length and the Dirichlet integral $D(u)$ are conformal invariants.

Let $p$ be a puncture on $S$. A collar about $p$ is a doubly connected domain in $S$ bounded by $p$ and a Jordan curve (called the boundary curve of the collar) orthogonal to the pencil of geodesics emanating from $p$. A collar about $p$ of area $\beta$ will be called a $\beta$-collar: $C_{\beta}(p)$.

Lemma 1.4. If $p$ is a puncture then given $\beta \leq 1$ and $\varepsilon>0$, there exists $\alpha=\alpha(\beta, \varepsilon)$ such that the harmonic function in $C_{\beta}(p) \backslash C_{\alpha}(p)$ which is 1 on the boundary curve of $C_{\beta}(p)$ and 0 on the boundary curve of $C_{\alpha}(p)$, satisfies

$$
\iint_{C_{\beta}(p) \backslash C_{\alpha}(p)}|\nabla v|^{2} \leq \varepsilon .
$$

Proof. Represent $S$ as $U / \Gamma$ and assume, since this can be achieved by conjugation, that $\Gamma$ contains a primitive element $z \rightarrow z+1$, and that the canonical map $\pi: U \rightarrow U / \Gamma=S$ takes vertical lines in $U$ into geodesics emanating from 
$p$. This implies that a collar about $p$ is the image under $\pi$ of a region $0 \leq x<1$, $y>\eta$ (for some $\eta>0$ ) on which $\pi$ is injective. One computes at once that, for a $\beta$-collar, $\eta=1 / \beta$.

It is known (see $[\mathrm{Kr}, \mathrm{p} .60-61]$ ) that there is a $\beta$-collar for every $\beta \leq 1$.

We lift $v$ to a function $w$ in $U$ :

$$
w(x+i y)=\left(\frac{1}{\alpha}-y\right) /\left(\frac{1}{\alpha}-\frac{1}{\beta}\right) .
$$

Then

$$
\iint_{C_{\beta}(p) \backslash C_{\alpha}(p)}|\nabla v|^{2}=\int_{0}^{1} \int_{1 / \beta}^{1 / \alpha}|\nabla w|^{2} d y d x=\frac{1}{1 / \alpha-1 / \beta}=\varepsilon
$$

if $\alpha=1 /(1 / \beta+1 / \varepsilon)$.

Now we start the proof of Theorem 2 . We assume that $b(S)>0$. Let $G$ be a geodesic domain in $S$. Let $\gamma_{j}$ be the proper closed simple geodesics bounding $G, j=1, \ldots, n$ and let $p_{i}$ be the punctures "surrounded" by $G, i=1, \ldots, m$. Fix a positive constant $d$.

Let $\Omega_{j}$ be $\Omega_{j}=\operatorname{int}\left\{p \in S \backslash G: d_{S}\left(p, \gamma_{j}\right) \leq d\right\}$.

Let $H_{k}$ be the connected component of $\cup_{j=1}^{n} \Omega_{j}$ which contains $\Omega_{k}$. To simplify notation we assume that $H_{k}=\Omega_{1} \cup \Omega_{2} \cup \cdots \cup \Omega_{k}$. Let $u_{k}$ be the harmonic function in $H_{k}$ such that $u=1$ in $\gamma_{1} \cup \cdots \cup \gamma_{k}$ and $u=0$ in $E_{k}=\partial H_{k} \backslash \cup_{1}^{k} \gamma_{j}$.

Denote by $\Gamma$ the collection of all curves in $H_{k}$ which join $E_{k}$ to $\gamma_{1} \cup \cdots \cup \gamma_{k}$.

The theorem in $[\mathrm{A}-\mathrm{S}]$ gives

$$
\frac{1}{\iint_{H_{k}}\left|\nabla u_{k}\right|^{2} d A_{S}}=\sup _{\rho} \frac{L(\Gamma, \rho)^{2}}{A\left(H_{k}, \rho\right)} \geq \frac{L\left(\Gamma, \rho_{S}\right)^{2}}{A\left(H_{k}, \rho_{S}\right)}=\frac{d^{2}}{A_{S}\left(H_{k}\right)}
$$

and using Lemma 1.3 we obtain

$$
\begin{aligned}
\iint_{H_{k}}\left|\nabla u_{k}\right|^{2} d A_{S} & \leq \frac{A_{S}\left(H_{k}\right)}{d^{2}} \leq \frac{A_{S}\left(\Omega_{1}\right)+\cdots+A_{S}\left(\Omega_{k}\right)}{d^{2}} \\
& \leq \frac{\sinh (d)}{d^{2}}\left[L_{S}\left(\gamma_{1}\right)+\cdots+L_{S}\left(\gamma_{k}\right)\right] .
\end{aligned}
$$

$f(d)=\sinh (d) / d^{2} \geq f\left(d_{0}\right)$ where $d_{0}=1.91500806 \ldots$ and $f_{0}=f\left(d_{0}\right)<0.9053$. Of course, we choose $d=d_{0}$.

We want to use

$$
0<b(S) \leq \frac{\iint|\nabla \varphi|^{2}}{\iint \varphi}
$$

for some $\varphi$ with compact support. 
We would like to define $\varphi$ as follows

$$
\varphi \equiv \begin{cases}1 & \text { in } G \\ u_{k} & \text { in } H_{k} \\ 0 & \text { elsewhere }\end{cases}
$$

but $\varphi$ must be 0 in a neighborhood of the punctures; so we will have to define $\varphi$ around the punctures in a different way. We do this as follows.

There exists $\beta_{0}$ such that $C_{\beta_{0}}\left(p_{i}\right) \subset G$ for all $i=1, \ldots, m$ and $C_{\beta_{0}}\left(p_{i}\right) \cap$ $C_{\beta_{0}}\left(p_{j}\right)=\emptyset$ if $i \neq j$.

If $\varepsilon \in\left(0, m \beta_{0}\right)$, then $C_{\varepsilon / m}\left(p_{i}\right)$ is contained in $G$ and $A_{S}\left(\cup_{1}^{m} C_{\varepsilon / m}\left(p_{i}\right)\right)=\varepsilon$.

We use Lemma 1.4 with $\beta=\varepsilon / m, \alpha=\beta / 2=\varepsilon / 2 m$, and let $v_{i}$ be as in the lemma and $v_{i} \equiv 0$ in $C_{\alpha}\left(p_{i}\right)$.

Then

$$
\iint_{C_{\beta}\left(p_{i}\right)}\left|\nabla v_{i}\right|^{2}=\frac{\varepsilon}{m} .
$$

If

$$
\varphi \equiv \begin{cases}1 & \text { in } G \backslash \cup_{1}^{m} C_{\beta}\left(p_{i}\right) \\ v_{i} & \text { in } C_{\beta}\left(p_{i}\right) \\ u_{k} & \text { in } H_{k} \\ 0 & \text { elsewhere }\end{cases}
$$

then

$$
b(S) \leq \frac{\iint|\nabla \varphi|^{2}}{\iint \varphi^{2}} \leq \frac{\varepsilon+f_{0} L_{S}(\partial G)}{A_{S}(G)-\varepsilon}
$$

for all $\varepsilon<m \beta_{0}$. Therefore

$$
b(S) \leq f_{0} \frac{L_{S}(\partial G)}{A_{S}(G)}
$$

and (1.4) implies that

$$
h(S) \leq 2+\frac{f_{0}}{b(S)},
$$

and since $b \leq \frac{1}{4}$, we obtain that

$$
h(S) b(S) \leq \frac{1}{2}+f_{0} .
$$

Therefore

$$
h(S) b(S)<\frac{3}{2} .
$$




\section{Proof of Theorem 1}

We assume that $f: S_{1} \rightarrow S_{2}$ is a $k$-quasiconformal mapping from the Riemann surface $S_{1}$ onto the Riemann surface $S_{2}$.

We shall need the following lemma which is certainly known.

Lemma 2.1. Let $\alpha_{1}$ be a closed simple curve on $S_{1}$ and let $\alpha_{2}=f\left(\alpha_{1}\right)$. Denote by $a_{i}$ the infimum of the lengths of all closed curves freely homotopic to $\alpha_{i}$ in $S_{i}$. Then

$$
\frac{1}{k} a_{2} \leq a_{1} \leq k a_{2} \text {. }
$$

Proof. If $a_{i}$ is 0 then either $\alpha_{i}$ is homotopic to zero or "surrounds" a puncture. Therefore the $a_{i}$ 's are zero simultaneously.

So we may assume that $a_{1} a_{2}>0$.

Let $A_{i}$ be a Möbius transformation from $\Delta$ onto $\Delta$ representing $\alpha_{i}, i=1,2$. Then the $A_{i}$ 's are hyperbolic, $a_{i}=$ translation length of $A_{i}=\inf _{z \in \Delta} d_{\Delta}\left(z, A_{i} z\right)$, and $A_{1}$ and $A_{2}$ are conjugates by a lift $\tilde{f}$ of $f(\tilde{f}: \Delta \rightarrow \Delta)$.

Let $\Omega_{i}$ be the quotient Riemann surface $\Delta /\left\langle A_{i}\right\rangle, i=1,2$. Then $\Omega_{i}$ is a ring whose modulus is $\pi / a_{i}$. Therefore

$$
\frac{\pi}{a_{2}} \leq k \frac{\pi}{a_{1}} .
$$

We assume now that $S_{2}$ satisfies HII and we shall check that $S_{1}$ must satisfy HII. Because of Lemma 1.2 it is enough to check that there exists a constant $C_{1}$ so that if $\Omega_{1}$ is a geodesic domain in $S_{1}$, then

$$
A_{S_{1}}\left(\Omega_{1}\right) \leq C_{1} L_{S_{1}}\left(\partial \Omega_{1}\right) \text {. }
$$

Let the boundary curves of $\Omega_{1}$ be denoted by $\alpha_{j}, j=1, \ldots, n$ (recall that $\alpha_{j}$ could be a puncture). Let $\beta_{j}, j=1, \ldots, n$ be the shortest curve in the free homotopy class of $f\left(\alpha_{j}\right)$. Thus $\beta_{j}$ is a closed simple geodesic. Let $\Phi$ be the domain bounded by the $\beta_{j}$ 's. Then $A_{S_{2}}(\Phi)=A_{S_{1}}\left(\Omega_{1}\right)$, because of the GaussBonnet theorem. But

$$
A_{S_{2}}(\Phi) \leq h_{2} \sum_{j=1}^{n} L_{S_{2}}\left(\beta_{j}\right) \leq h_{2} k L_{S_{1}}\left(\partial \Omega_{1}\right)
$$

and so we see that $S_{1}$ satisfies HII with a constant $h_{1}$ satisfying

$$
h_{1} \leq 2+h_{2} k \leq 3 h_{2} k \text {. }
$$

In particular we have seen that

$$
b\left(S_{1}\right)^{2} \leq C k^{2} b\left(S_{2}\right)
$$

where $C \leq 36\left(\frac{1}{2}+f_{0}\right)^{2}<72$. 


\section{Proof of Theorem 3}

Let us denote by $t$ the $\inf _{n \neq m} d_{G}\left(a_{n}, a_{m}\right)$.

Instead of showing that $b(\hat{G})>0$, we will prove that $h(\hat{G}) \leq C$.

In this section, by $C$ we will mean a constant which depends only on $t$ and the modulus of $G$.

Let $I$ be $I=\left\{a_{n}\right\}_{n=1}^{\infty}$.

The proof is easy if $I=\emptyset$ :

In $[\mathrm{O}]$ it is proved that

$$
\left|\nabla \log \lambda_{G}(z)\right| \leq \frac{2}{d(z, \partial G)}
$$

Since $G$ is modulated, there is $C_{0}>0$ such that

$$
\lambda_{G}(z) \geq \frac{C_{0}}{d(z, \partial G)}
$$

Therefore $\left|\nabla \log \lambda_{G}(z)\right| \leq C \lambda_{G}(z)$.

We have $\lambda_{G}^{2}=\Delta \log \lambda_{G}$ because the metric $\lambda_{G}|d z|$ has Gaussian curvature -1 . If $D$ belongs to $\mathcal{D}(G)$, then, using Green's formula,

$$
A_{G}(D)=\iint_{D} \lambda_{G}^{2}(z) d x d y=\iint_{D} \Delta \log \lambda_{G}(z) d x d y=\int_{\partial D} \nabla \log \lambda_{G}(z) \cdot \vec{n}|d z|
$$

where $\vec{n}$ is the unit outer normal of $\partial D$.

And so

$$
A_{G}(D) \leq \int_{\partial D}\left|\nabla \log \lambda_{G}(z)\right||d z| \leq C \int_{\partial D} \lambda_{G}(z)|d z|=C L_{G}(\partial D) .
$$

Therefore $G$ has HII with $h(G) \leq 2 / C_{0}$.

The idea of the proof of Theorem 3 is to divide $G$ into two pieces: the first piece will be "far" from $I$ and then $\lambda_{\hat{G}} \sim \lambda_{G}$; the second part will be "around" $I$ and then $\lambda_{\hat{G}} \sim \lambda_{\Delta^{*}}$. We will choose the neigborhood of $I$ so that the constants appearing in the estimates will be independent of the neighborhoods.

Lemma 3.1. If $S \equiv G \backslash \cup_{n \geq 1} \Delta\left(a_{n}, \frac{1}{2} \varepsilon d\left(a_{n}, \partial G\right)\right)$ with $\varepsilon>0$, then

$$
\lambda_{G}(z) \leq \lambda_{\hat{G}}(z) \leq C \lambda_{G}(z)
$$

for all $z \in S$. 
Proof. Since $\hat{G} \subset G, \lambda_{G}(z) \leq \lambda_{\hat{G}}(z)$ for all $z \in \hat{G}$.

Let $z \in S$. It is easy to check that

$$
|z-a| \geq \frac{\varepsilon}{2+\varepsilon} d(z, \partial G), \quad \text { for all } a \in I .
$$

Therefore $d(z, \partial \hat{G})=d(z, I \cup \partial G) \geq \varepsilon /(2+\varepsilon) d(z, \partial G)$.

And so, because of Schwarz's lemma, we finally get

$$
\lambda_{\hat{G}}(z) \leq \frac{2}{d(z, \hat{G})} \leq \frac{2(2+\varepsilon)}{\varepsilon d(z, G)} \leq \frac{2(2+\varepsilon)}{2 C_{0}} \lambda_{G}(z) .
$$

Lemma 3.2. The disks $\left\{\Delta\left(a_{n}, \varepsilon d\left(a_{n}, \partial G\right)\right)\right\}_{n \geq 1}$ are disjoint if $\varepsilon=t /(4+2 t)$. Furthermore, if $K_{1} \equiv e^{K} \equiv(3+2 \sqrt{2}) e^{4}$ and $z \in \bar{\Delta}(a, \varepsilon d(a, \partial G))$ where $a \in I$, then

$$
\frac{1}{\sqrt{2}} \lambda_{\Delta\left(a, K_{1} d(a, \partial G)\right)^{*}}(z) \leq \lambda_{\hat{G}}(z) \leq \lambda_{\Delta(a, 2 \varepsilon d(a, \partial G))^{*}}(z) .
$$

Proof. Assume that the disks are not disjoint. Then there are $m, n,(m \neq n)$ such that

$$
\left|a_{n}-a_{m}\right|<2 \varepsilon d\left(a_{n}, \partial G\right) .
$$

Let $\gamma$ be the line segment which joins $a_{n}$ with $a_{m}$; if $z \in \gamma$

$$
d\left(a_{n}, \partial G\right) \leq\left|a_{n}-z\right|+d(z, \partial G)<2 \varepsilon d\left(a_{n}, \partial G\right)+d(z, \partial G) .
$$

and then

$$
d\left(a_{n}, \partial G\right)<\frac{1}{1-2 \varepsilon} d(z, \partial G)
$$

It follows that

$$
d_{G}\left(a_{n}, a_{m}\right) \leq \int_{\gamma} \lambda_{G}(z)|d z| \leq \int_{\gamma} \frac{2|d z|}{d(z, \partial G)} \leq \frac{2}{1-2 \varepsilon} \int_{\gamma} \frac{|d z|}{d\left(a_{n}, \partial G\right)}
$$

and so

$$
d_{G}\left(a_{n}, a_{m}\right) \leq \frac{2\left|a_{n}-a_{m}\right|}{(1-2 \varepsilon) d\left(a_{n}, \partial G\right)}<\frac{4 \varepsilon}{1-2 \varepsilon}=t,
$$

which contradicts the definition of $t$.

Therefore, the disks $\left\{\Delta\left(a_{n}, \varepsilon d\left(a_{n}, \partial G\right)\right)\right\}_{n \geq 1}$ are disjoint.

The second inequality is then obvious.

The first inequality follows from Theorem 1 of [B-P].

Now we start the proof of Theorem 3 .

Let $D$ be a domain which is relatively compact in $\hat{G}$ with $\partial D=\beta \cup \beta_{1} \cup$ $\cdots \cup \beta_{k} \cup \beta^{1} \cup \cdots \cup \beta^{l}$. 
The $\beta, \beta_{j}, \beta^{i}$ are Jordan curves. We may assume that they are disjoint. Also, $\beta$ is the outer connected component of $\partial D$, the $\beta_{j}$ are not homotopic to zero in $G$, and the $\beta^{i}$ are homotopic to zero in $G$, with $\beta^{i}=\partial B^{i}$ where $B^{i}$ is a closed Jordan domain in $G$.

If $B=D \cup\left\{\cup_{i=1}^{l} B^{i}\right\} \backslash I$ then the boundary curves of $B$ are $\beta \cup \beta_{1} \cup \cdots \cup \beta_{k}$. It is enough to show that

$$
A_{\hat{G}}(B) \leq C L_{\hat{G}}(\partial B)
$$

since we have increased the area and decreased the length.

Let $d_{n} \equiv d\left(a_{n}, \partial G\right)$ and define $I_{B} \equiv\left\{a_{n}: B \cap \Delta\left(a_{n}, \frac{1}{2} \varepsilon d_{n}\right) \neq \emptyset\right\}$,

$I_{1} \equiv\left\{a_{n}: \partial B \cap \Delta\left(a_{n}, \frac{1}{2} \varepsilon d_{n}\right)^{*} \neq \emptyset\right\}$ and $I_{2} \equiv\left\{a_{n}: \Delta\left(a_{n}, \frac{1}{2} \varepsilon d_{n}\right)^{*} \subset B\right\}$.

Observe that $I_{1} \cap I_{2}=\emptyset$ and $I_{1} \cup I_{2}=I_{B}$.

Lemma 3.2 implies that

$$
\begin{aligned}
d_{\hat{G}}\left(\left\{\left|z-a_{n}\right|\right.\right. & \left.\left.=\frac{1}{2} \varepsilon d_{n}\right\},\left\{\left|z-a_{n}\right|=\varepsilon d_{n}\right\}\right) \geq \frac{1}{\sqrt{2}} d_{\Delta\left(a_{n}, K_{1} d_{n}\right)^{*}}(\{\},\{\}) \\
& =\frac{1}{\sqrt{2}} \log \frac{\log \left(2 k_{1} / \varepsilon\right)}{\log \left(k_{1} / \varepsilon\right)} \equiv \alpha .
\end{aligned}
$$

We distinguish two cases.

Case 1. $L_{\hat{G}}\left(\partial B \cap \Delta\left(a_{n}, \varepsilon d_{n}\right)^{*}\right)<\alpha$ for some $a_{n} \in I_{1}$.

Since $d_{\hat{G}}\left(\left\{\left|z-a_{n}\right|=\frac{1}{2} \varepsilon d_{n}\right\},\left\{\left|z-a_{n}\right|=\varepsilon d_{n}\right\}\right) \geq \alpha, \partial B \cap \Delta\left(a_{n}, \varepsilon d_{n}\right)^{*}$ contains a boundary curve $\gamma$ of $B$. Then $\gamma$ is a $\beta_{j}$ or $\gamma=\beta$. Actually, $\gamma$ can not be a $\beta_{j}$ since $\gamma \subset \Delta\left(a_{n}, \varepsilon d_{n}\right)$ and so it is homotopic to zero in $G$. So $\gamma=\beta$ and we conclude that $B \subset \Delta\left(a_{n}, \varepsilon d_{n}\right)^{*}$ and $B$ is simply or doubly connected. By Lemma 1.1

$$
A_{\hat{G}}(B) \leq L_{\hat{G}}(\partial B) .
$$

Case 2. $L_{\hat{G}}\left(\partial B \cap \Delta\left(a_{n}, \varepsilon d_{n}\right)^{*}\right) \geq \alpha$ for all $a_{n} \in I_{1}$ or $I_{1}=\emptyset$.

We have

$$
A_{\hat{G}}(B) \leq A_{\hat{G}}(B \cap S)+\sum_{a_{n} \in I_{2}} A_{\hat{G}}\left(\Delta\left(a_{n}, \frac{1}{2} \varepsilon d_{n}\right)^{*}\right)+\sum_{a_{n} \in I_{1}} A_{\hat{G}}\left(\Delta\left(a_{n}, \frac{1}{2} \varepsilon d_{n}\right)^{*}\right)
$$

We will treat each summand separately.

First, $A_{\hat{G}}(B \cap S) \leq C A_{G}(B \cap S)$ because of Lemma 3.1.

Seccnd, $A_{\hat{G}}\left(\Delta\left(a_{n}, \frac{1}{2} \varepsilon d_{n}\right)^{*}\right) \leq C A_{G}\left(\Delta\left(a_{n}, \frac{1}{2} \varepsilon d_{n}\right)^{*}\right)$ because of Lemma 3.2.

Then

$$
\begin{aligned}
A_{\hat{G}}(B \cap S)+ & \sum_{a_{n} \in I_{2}} A_{\hat{G}}\left(\Delta\left(a_{n}, \frac{1}{2} \varepsilon d_{n}\right)^{*}\right) \\
& \leq C A_{G}(B \cap S)+C \sum_{a_{n} \in I_{2}} A_{G}\left(\Delta\left(a_{n}, \frac{1}{2} \varepsilon d_{n}\right)^{*}\right) \\
& \leq C A_{G}(B) \leq C L_{G}(\partial B) \leq C L_{\hat{G}}(\partial B) .
\end{aligned}
$$


Finally, we deal with the last term. We have

$$
A_{\hat{G}}\left(\Delta\left(a_{n}, \frac{1}{2} \varepsilon d_{n}\right)^{*}\right) \leq C
$$

because of Lemma 3.2.

Since $a_{n} \in I_{1}$, we have that

$$
A_{\hat{G}}\left(\Delta\left(a_{n}, \frac{1}{2} \varepsilon d_{n}\right)^{*}\right) \leq \frac{C}{\alpha} L_{\hat{G}}\left(\partial B \cap \Delta\left(a_{n}, \varepsilon d_{n}\right)^{*}\right) .
$$

and so

$$
\sum_{a_{n} \in I_{1}} A_{\hat{G}}\left(\Delta\left(a_{n}, \frac{1}{2} \varepsilon d_{n}\right)^{*}\right) \leq C \sum_{a_{n} \in I_{1}} L_{\hat{G}}\left(\partial B \cap \Delta\left(a_{n}, \varepsilon d_{n}\right)^{*}\right) \leq C L_{\hat{G}}(\partial B) .
$$

because the disks $\left\{\Delta\left(a_{n}, \varepsilon d_{n}\right)\right\}_{n \geq 1}$ are disjoint.

And so (3.1), (3.2) and (3.3) give

$$
A_{\hat{G}}(B) \leq C L_{\hat{G}}(\partial B) .
$$

\section{Proof of Theorem 4}

Lemma 4.1. Let $E$ be a compact set, $E \subset \bar{\Delta}$, with $\{0,1\} \subset E$ and cap $E \leq \varepsilon$. There is a universal constant $C$ such that if $R>2$ then

$$
b\left(\Delta_{R} \backslash E\right) \leq C\left(\frac{1}{\log (1 / \varepsilon)}+\frac{1}{\log \frac{1}{2} R}\right)
$$

Proof. We can find an open set $A$ with $C^{\infty}$-boundary and containing $E$, such that if we let $u$ be the harmonic function in $\Delta_{2} \backslash \bar{A}$ with boundary values

$$
\begin{cases}u=0 & \text { in } \partial A \\ u=1 & \text { in }|z|=2\end{cases}
$$

then

$$
\iint|\nabla u|^{2} \leq \frac{C}{\log (1 / \varepsilon)} \quad \text { and } \quad u(z) \geq \frac{1}{2} \quad \text { in }|z| \geq \frac{3}{2},
$$

where $C$ is an absolute constant.

Let $R^{\prime}$ be a positive number less than $R$.

Define a function $V$ on $\Delta_{R} \backslash E$ as follows

$$
V \equiv \begin{cases}u & \text { in } \Delta_{2} \backslash A \\ 1-\log \frac{1}{2}|z| / \log \frac{1}{2} R^{\prime} & \text { in } 2 \leq|z| \leq R^{\prime} \\ 0 & \text { elsewhere }\end{cases}
$$


Clearly

$$
|\nabla V|^{2}= \begin{cases}|\nabla u|^{2} & \text { in } \Delta_{2} \backslash A \\ 1 /|z|^{2}\left(\log \frac{1}{2} R^{\prime}\right)^{2} & \text { in } 2<|z|<R^{\prime} \\ 0 & \text { elsewhere. }\end{cases}
$$

We have that

$$
\begin{gathered}
\iint_{\Delta_{R} \backslash E}|\nabla V|^{2}=\iint_{\Delta_{2} \backslash A}|\nabla u|^{2}+\iint_{2 \leq|z| \leq R^{\prime}} \frac{1}{|z|^{2}\left(\log \frac{1}{2} R^{\prime}\right)^{2}} \\
\iint_{\Delta_{R} \backslash E}|\nabla V|^{2} \leq \frac{C}{\log (1 / \varepsilon)}+\frac{2 \pi}{\log \frac{1}{2} R^{\prime}},
\end{gathered}
$$

and also

$$
\begin{gathered}
\iint_{\Delta_{R} \backslash E} V^{2} \lambda_{\Delta_{R} \backslash E}^{2} \geq \iint_{3 / 2 \leq|z| \leq 2} u^{2} \lambda_{\Delta_{R} \backslash E}^{2} \geq \frac{1}{4} A_{\Delta_{R} \backslash E}\left(\left\{\frac{3}{2} \leq|z| \leq 2\right\}\right) \\
\iint_{\Delta_{R} \backslash E} V^{2} \lambda_{\Delta_{R} \backslash E}^{2} \geq \frac{1}{4} A_{C \backslash\{0,1\}}\left(\left\{\frac{3}{2} \leq|z| \leq 2\right\}\right) \equiv C .
\end{gathered}
$$

Therefore

$$
b\left(\Delta_{R} \backslash E\right) \leq \frac{\iint|\nabla V|^{2}}{\iint V^{2} \lambda^{2}} \leq C\left(\frac{1}{\log (1 / \varepsilon)}+\frac{1}{\log \frac{1}{2} R^{\prime}}\right)
$$

for each $R^{\prime}<R$, and so

$$
b\left(\Delta_{R} \backslash E\right) \leq C\left(\frac{1}{\log (1 / \varepsilon)}+\frac{1}{\log \frac{1}{2} R}\right) .
$$

We deduce the following consequences:

Corollary 1. Let $E$ be a compact set contained in $\bar{\Delta}_{r}$, where $0<r<1$. Assume that $\{0, r\} \subset E$ and also that $\operatorname{cap} E / r \leq \varepsilon$. Then

$$
b(\Delta \backslash E) \leq C\left(\frac{1}{\log (1 / \varepsilon)}+\frac{1}{\log (1 / 2 r)}\right) .
$$

Corollary 2. Let $E$ be a compact set contained in $\bar{\Delta}_{r}$, where $0<r<1$. Assume that 0 belongs to $E, E \neq\{0\}$ and $\operatorname{cap} E=0$. Then

$$
\delta(\Delta \backslash E) \geq 1-\frac{C}{\log (1 / 2 r)} \quad \text { if } r<r_{0} .
$$

Corollary 3. If $a, b \in \Delta$, then

$$
\delta(\Delta \backslash\{a, b\}) \geq 1-h\left(d_{\Delta}(a, b)\right) \quad \text { if } d_{\Delta}(a, b)<d_{0} .
$$

where $h(t)=C / \log \left(\frac{1}{2} \operatorname{cotanh} \frac{1}{2} t\right)$. 
This is proved in [F2] without the explicit expression for $h$.

The following lemma will be frequently used. It will allow us to glue parts of the boundary while keeping $\delta$ under control.

Lemma 4.2. Let $\Omega$ be a domain in the complex plane. If $G$ is a connected closed set such that $G \cap \partial \Omega \neq \emptyset$ and $\hat{\Omega}$ is a connected component of $\Omega \backslash G$, then

$$
\delta(\Omega) \geq \delta(\hat{\Omega})
$$

Proof. In $\hat{\Omega}$ there are fewer curves and they are longer. See [Ca, Theorem 5.1].

Lemma 4.3. Let $E$ be an infinite compact set contained in the plane domain $\Omega$. Assume that cap $E=0$. Then

$$
\delta(\Omega \backslash E)=1
$$

We should think of $\Omega$ as being $\Delta$.

Proof. The theorem of Cantor-Bendixson (see, for instance, [Ku, p. 183]) implies that $E=P \cup R$ where $P$ is the maximal perfect subset of $E$ and $R$ is a countable set.

We distinguish two cases.

Case (A): $P=\emptyset$. Then $E=R$ is a closed countable set.

Write $E=E^{d} \cup E^{i}$. Let us see first that $E^{i}$ and $E^{d}$ are non-empty.

If $E^{i}=\emptyset$, then $E\left(=E^{d}\right)$ is a perfect set. It follows that $E \subset P=\emptyset$ which contradicts that $E$ is infinite.

If $E^{d}=\emptyset$, then $E\left(=E^{i}\right)$ is a compact discrete set. If follows that $E$ is finite which contradicts our hypothesis.

Now, $E^{d}=\left(E^{d}\right)^{d} \cup\left(E^{d}\right)^{i}$. Notice that $\left(E^{d}\right)^{i}$ is non-empty, for if $\left(E^{d}\right)^{i}=\emptyset$, then $E^{d}\left(=\left(E^{d}\right)^{d}\right)$ is a perfect set. It follows that $E^{d} \subset P=\emptyset$ contradicting that $E^{d} \neq \emptyset$.

If $e$ belongs to $\left(E^{d}\right)^{i}$, there is $r>0$ such that $\Delta(e, 2 r) \cap E^{d}=\{e\}$. Since $e$ belongs to $E^{d}$, we have that $\Delta(e, r) \cap E=\{e\} \cup\left\{a_{n}\right\}_{n}$ with $a_{n} \in E^{i}$ and $a_{n} \rightarrow e$.

With the notation of Lemma 4.2 and setting $G=\Delta(e, r)^{c}$ and $B=\{e\} \cup$ $\left\{a_{n}\right\}_{n}$, we see that

$$
\delta(\Omega \backslash E) \geq \delta(\Delta(e, r) \backslash B)
$$

In [Pa, Theorem 4.1] it is proved that if $S$ is a hyperbolic Riemann surface and $A$ is a discrete subset of $S$, then

$$
\delta(S \backslash A) \geq \delta(S)
$$

It follows that

$$
\delta(\Delta(e, r) \backslash B) \geq \delta\left(\Delta(e, r) \backslash\left\{e, a_{n}\right\}\right)=\delta\left(\Delta \backslash\left\{0, \frac{a_{n}-e}{r}\right\}\right) .
$$


Corollary 2 implies that

$$
\delta\left(\Delta \backslash\left\{0, \frac{a_{n}-e}{r}\right\}\right) \geq 1-\frac{C}{\log \left(r / 2\left|a_{n}-e\right|\right)} .
$$

Then

$$
\delta(\Omega \backslash E) \geq 1-\frac{C}{\log \left(r / 2\left|a_{n}-e\right|\right)},
$$

for each $n$.

And so the lemma is proved in this case.

Case (B): $P \neq \emptyset$.

The set $P$ is not uniformly perfect because cap $P=0$. It follows that if $k$ is a natural number greater than 1 , then there must be a point $a_{k}$ in $P$ and a positive number $r_{k}$ such that $B_{k} \cap P=\emptyset$, where $B_{k}$ is the ring

$$
\left\{z: \frac{r_{k}}{k} \leq\left|z-a_{k}\right| \leq k r_{k}\right\} \subset \Omega .
$$

Notice that $E \cap B_{k}\left(=R \cap B_{k}\right)$ is a closed countable set. It follows that there are $s_{k}, t_{k}$, with $s_{k} \in\left[r_{k} / k, r_{k} / \sqrt{k}\right], t_{k} \in\left[\sqrt{k} r_{k}, k r_{k}\right]$, such that

$$
\left\{\left|z-a_{k}\right|=s_{k}\right\} \cap E=\emptyset \quad \text { and } \quad\left\{\left|z-a_{k}\right|=t_{k}\right\} \cap E=\emptyset .
$$

If $A_{k}$ is the ring $\left\{z: s_{k} \leq\left|z-a_{k}\right| \leq t_{k}\right\}$, then $A_{k} \cap P=\emptyset$ and $A_{k} \cap E(=$ $\left.A_{k} \cap R\right)$ is a compact set contained in the interior of $A_{k}$.

If $A_{k} \cap E$ is infinite for some $k$, then we are in case (A).

So we may assume that $A_{k} \cap E$ is finite for all $k>1$. Lemma 4.2 implies

$$
\delta(\Omega \backslash E) \geq \delta\left(\Delta\left(a_{k}, t_{k}\right) \backslash E\right) .
$$

Applying Patterson's theorem again, we obtain

$$
\delta(\Omega \backslash E) \geq \delta\left(\Delta\left(a_{k}, t_{k}\right) \backslash\left\{E \cap \Delta\left(a_{k}, s_{k}\right)\right\}\right) \geq 1-\frac{C}{\log \left(t_{k} / 2 s_{k}\right)},
$$

therefore

$$
\delta(\Omega \backslash E) \geq 1-\frac{C}{\log \frac{1}{2} k} \quad \text { for all } k>1
$$

and so

$$
\delta(\Omega \backslash E)=1 .
$$

Now we start the proof of Theorem 4.

Let us define $P=\hat{C} \backslash H \backslash I$.

Let us observe that $P$ is not empty. 
If $P$ were empty then $H$ would be the Riemann sphere minus finitely many points, but this is ruled out since $\delta(H)<1$.

Moreover, we will see that

$$
\operatorname{cap}(\Delta(p, r) \cap P)>0 \quad \text { for each } p \in P \quad \text { and } r>0 .
$$

Assume not, then there exist $p \in P$ and $r>0$ such that $\operatorname{cap}(\Delta(p, r) \cap P)=0$.

We may assume that $p=0$ and $r=1$, and then $\operatorname{cap}(\Delta \cap P)=0$.

Let $R$ be the set $\{s \in(0,1):\{|z|=s\} \cap(P \cup I) \neq \emptyset\}$. R is a closed set in $(0,1)$ and $\operatorname{cap} R=0$. Therefore there are $s \in(0,1)$ and $\varepsilon>0$ such that $R \cap[s-\varepsilon, s]=\emptyset$.

Then the set $E$ defined as $E=\Delta_{s} \cap(P \cup I)=\Delta_{s-\varepsilon} \cap(P \cup I)$ is a compact set in $\Delta_{s}$ and $\operatorname{cap} E=0$. Notice that $E$ is infinite because $p \notin I$.

Therefore, using Lemma 4.2 and Lemma 4.3 we deduce that

$$
\delta(H) \geq \delta\left(\Delta_{s} \backslash E\right)=1
$$

which contradicts $b(H)>0$.

We conclude that $\operatorname{cap}(\Delta(p, r) \cap P)>0$ for each $p \in P$, for each $r>0$.

Next we check that the points of $I$ are separated in $\hat{\mathbf{C}} \backslash P$. Fix a point $q$ of $H$.

Let $F$ be a universal covering map $F: \Delta \rightarrow \hat{\mathbf{C}} \backslash P$ such that $F(0)=q$.

Define $J \equiv F^{-1}(I)$ and let $G$ be a universal covering map $G: \Delta \rightarrow \Delta \backslash J$ such that $G(0)=0$.

Then $\Pi \equiv F \circ G: \Delta \rightarrow H$ is a universal covering map of $H$ and $\Pi(0)=q$.

Let $\gamma$ be an isometry of $\Delta$. If $G \circ \gamma=G$, then $\Pi \circ \gamma=\Pi$.

It follows that the group $\mathcal{G}(G)$ of covering transformations of $G$ forms a subgroup of $\mathcal{G}(\Pi)$, the covering transformations of $\Pi$. Therefore

$$
\sum_{\gamma \in \mathcal{G}(G)}\left(\frac{1-|\gamma(0)|}{1+|\gamma(0)|}\right)^{t} \leq \sum_{\gamma \in \mathcal{G}(\Pi)}\left(\frac{1-|\gamma(0)|}{1+|\gamma(0)|}\right)^{t}
$$

and so $\delta(\Delta \backslash J) \leq \delta(H)<1$.

If $i, j$ belong to $I$, then there are $k, l$ belonging to $J$ such that $d_{\Delta}(k, l)=$ $d_{\hat{\mathbf{C}} \backslash P}(i, j)$. Then, using Patterson's theorem and Corollary 3

$$
\delta(H) \geq \delta(\Delta \backslash J) \geq \delta(\Delta \backslash\{k, l\}) \geq 1-h\left(d_{\Delta}(k, l)\right) .
$$

Therefore

$$
d_{\hat{\mathbf{C}} \backslash P}(i, j) \geq 2 \operatorname{Argtanh}\left(\frac{1}{2} \exp \left(\frac{-C}{1-\delta(H)}\right)\right) .
$$

Finally we check the behaviour of $\alpha$ and $\beta$. 
Fix $p \in P$ and $0<r<\frac{1}{2} \operatorname{diam} P$. Let $\beta=\beta(p, r)$.

Let $C_{1}$ be such that if $\beta<2 C_{1}$ then $C / \log (1 /(2 \beta))<\frac{1}{2} b(H)$. Also $C_{1}<\frac{1}{4}$. If $\beta<2 C_{1}$, then

$$
\bar{\Delta}(p, \beta r) \cap P=\Delta(p, r) \cap P, \quad \text { and } \beta r<\frac{1}{2} r .
$$

Since $\operatorname{cap}(\Delta(p, r) \cap P)>0$ (notice that $\Delta(p, r) \cap P$ has at least two points), we can use Lemma 4.1 :

$$
b(\Delta(p, r) \backslash P) \leq C\left(\frac{1}{\log (\beta r / \operatorname{cap}(P \cap \Delta(p, r)))}+\frac{1}{\log (1 /(2 \beta))}\right) .
$$

Now we apply Lemma 4.2 and Patterson's theorem to see that

$$
\delta(H) \geq \delta(H \cap \Delta(p, r))=\delta(\Delta(p, r) \backslash(P \cup I)) \geq \delta(\Delta(p, r) \backslash P)
$$

and so

$$
b(H) \leq b(\Delta(p, r) \backslash P)
$$

Therefore

$$
b(H)<\frac{C_{3}}{\log (\beta r / \operatorname{cap}(P \cap \Delta(p, r)))} .
$$

and then

$$
\frac{\operatorname{cap}(P \cap \Delta(p, r))}{\beta r}>\exp \left(\frac{-C_{3}}{b(H)}\right) \equiv C_{4} .
$$

It follows that

$$
\frac{\operatorname{cap}(\bar{\Delta}(p, \beta r) \cap P)}{\beta r}>C_{4}
$$

i.e.

$$
\alpha(p, r \beta(p, r))>C_{4} \text {. }
$$

Now we consider the case $\frac{1}{2} \operatorname{diam} P \leq r<\operatorname{diam} P$. $2 C_{1}$.

If $\beta(p, r)<C_{1}\left(<\frac{1}{4}\right)$ then $r \beta(p, r)=\frac{1}{2} r \beta\left(p, \frac{1}{2} r\right)$ and $\beta\left(p, \frac{1}{2} r\right)=2 \beta(p, r)<$

It follows that

$$
\frac{\operatorname{cap}(\bar{\Delta}(p, r \beta(p, r)) \cap P)}{r \beta(p, r)}=\frac{\operatorname{cap}\left(\bar{\Delta}\left(p, \frac{1}{2} r \beta\left(p, \frac{1}{2} r\right)\right) \cap P\right)}{\frac{1}{2} r \beta\left(p, \frac{1}{2} r\right)}>C_{2},
$$

i.e.

$$
\alpha(p, r \beta(p, r))>C_{2} .
$$

This finishes the proof.

Remark. In [F1] there is an example of a plane domain which is not bass while its boundary is not uniformily perfect but it is perfect. It is also easy to construct examples of bass plane domains which satisfy the conclusion of Theorem 4. It would be interesting to have euclidean criteria for deciding whether a plane domain is bass or not. 


\section{References}

[A-S] Ahlfors, L.V., and L. SARIo: Riemann surfaces. - Princeton University Press, Princeton, 1960.

[B-P] Beardon, A., and Ch. Pommerenke: The Poincaré metric of a plane domain. - J. London Math. Soc. (2) 18, 1978, 475-483.

[B] Buser, P.: A note on the isoperimetric constant. - Ann. Sci. École Norm. Sup. (4) 15, 1982, 213-230.

[Ca] Carleson, L.: On a class of meromorphic functions and its associated exceptional sets. - Thesis, Uppsala, 1950.

[Cha] Chavel, I.: Eigenvalues in Riemannian geometry. - Academic Press, New York, 1984.

[Che] Cheeger, J.: A lower bound for the smallest eigenvalue of the Laplacian. - In Problems in Analysis, Princeton University Press, Princeton, 1970, 195-199.

[F1] FernándeZ, J.L.: Domains with strong barrier. - Rev. Mat. Iberoamericana (to appear).

[F2] FERnÁNDEZ, J.L.: Singularities of inner functions. - Math. Z. 193, 1986, 393-396.

[Kr] KrA, I.: Automorphic forms and Kleinian groups. - W.A. Benjamin, Reading, 1972.

[Ku] Kuratowski, K.: Introduction to set theory and topology. - PWN-Polish Scientific Publishers, Warszawa, 1977.

[L] Lyons, T.: Instability of the Liouville property for quasi-isometric Riemannian manifolds and reversible Markov chains. - J. Diff. Geom. 26, 1987, 33-66.

[M] Minda, D.: Normal and Bloch functions in general planar domains. - Holomorphic functions and moduli, edited by D. Drasin, Springer-Verlag, New York, 1988.

[O] OsGood, B.G.: Some properties of $f^{\prime \prime} / f^{\prime}$ and the Poincaré metric. - Indiana Univ. Math. J. 31:4, 1982, 449-461.

[Pa] Patterson, S.J.: Some examples of Fuchsian groups. - Proc. London Math. Soc. (3) 39, 1979, 276-298.

[Pf] Pfluger, A.: Sur un propriété de l'application quasi-conforme d'une surface de Riemann ouverte. - C. R. Acad. Sci. Paris 227, 1948, 25-26.

[Po1] Pommerenke, Сh.: Uniformly perfect sets and the Poincaré metric. - Arch. Math. 32, $1979,192-199$.

[Po2] Pommerenke, Сh.: On uniformly perfect sets and Fuchsian groups. - Analysis 4, 1984, $299-321$.

[Ro] Royden, H.L.: A property of quasi-conformal mapping. - Proc. Amer. Math. Soc. 5, 1954, 266-269.

[S] Sullivan, D.: Related aspects of positivity in Riemannian geometry. - J. Differential Geom. 25, 1987, 327-351.

[T1] ToKI, Y.: On the classification of open Riemann surfaces. - Osaka J. Math. 4, 1952, 191-201.

[T2] ToKI, Y.: On the examples in the classification of open Riemann surfaces. - Osaka J. Math. 5, 1953, 267-280.

Universidad Autónoma de Madrid

Departamento de Matemáticas

E-28049 Madrid

Spain

Received 14 August 1989
Universidad Autónoma de Madrid

Departamento de Matemáticas E-28049 Madrid

Spain 